\title{
Mixed Integer Linear Programming for new trends in wind farm cable routing
}

\author{
Fischetti, Martina; Pisinger, David
}

Published in:

Electronic Notes in Discrete Mathematics

Link to article, DOI:

10.1016/j.endm.2018.01.013

Publication date:

2018

Document Version

Peer reviewed version

Link back to DTU Orbit

Citation (APA):

Fischetti, M., \& Pisinger, D. (2018). Mixed Integer Linear Programming for new trends in wind farm cable routing. Electronic Notes in Discrete Mathematics, 64, 115-124. https://doi.org/10.1016/j.endm.2018.01.013

\section{General rights}

Copyright and moral rights for the publications made accessible in the public portal are retained by the authors and/or other copyright owners and it is a condition of accessing publications that users recognise and abide by the legal requirements associated with these rights.

- Users may download and print one copy of any publication from the public portal for the purpose of private study or research.

- You may not further distribute the material or use it for any profit-making activity or commercial gain

- You may freely distribute the URL identifying the publication in the public portal

If you believe that this document breaches copyright please contact us providing details, and we will remove access to the work immediately and investigate your claim 


\title{
Mixed Integer Linear Programming for new trends in wind farm cable routing
}

\author{
Martina Fischetti ${ }^{1,2}$ \\ Vattenfall BA Wind and \\ Technical University of Denmark, Operations Research, \\ DTU Management Produktionstorvet, 426 B, DK-2800 Kgs. Lyngby, Denmark \\ David Pisinger ${ }^{3}$ \\ Technical University of Denmark, Operations Research, \\ DTU Management Produktionstorvet, 426 B, DK-2800 Kgs. Lyngby, Denmark
}

\begin{abstract}
The efficient production of green energy plays an import role in modern economies. In this paper we address the optimization of cable connections between turbines in an offshore wind park. Different versions of this problem have been studied recently. In a previous joint project with Vattenfall BA Wind (a global leader in energy production) we have studied and modeled the main constraints arising in practical cases. Building on that model, in the present paper we address new features that have been recently proposed by Vattenfall's experts. Turbines are becoming still more customized, therefore it is important to be able to evaluate the impact of new technologies with a flexible optimization tool. We here show how some new features can effectively be modeled and solved using a Mixed-Integer Linear Programming paradigm. Computational results on a real-world case are briefly presented.
\end{abstract}

Keywords: Network models, wind farm optimization, mixed-integer linear programming, computational analysis. 


\section{Introduction}

The production of green (in particular, wind) energy is an important topic both in industry and academia. As modern wind parks are getting bigger in size and in produced power, it is very important to optimize their design.

In this paper we address the optimization of cable connections among offshore turbines, called cable routing in what follows. Different versions of this problem have been studied in the recent literature, including $[2,3,4,5,7]$. Thanks to the collaboration between Vattenfall BA Wind (a global leader in energy production) and Technical University of Denmark (DTU), in the last couple of years we have been able to identify the constraints that arise in practical cases - some of which were missing in previous work from the literature. In particular, in [6] we introduced a new solution framework based on a Mixed-Integer Linear Programming (MILP) model, which is solved either exactly (through a commercial MILP solver) or heuristically (through an adhoc matheuristic scheme). The resulting optimization tool has been validated by Vattenfall's experts, and is routinely used by the planners. The tool is able to cope with practical needs such as the possibility of curvy cable connections, the need to avoid obstacles in the site, and power losses; see [6] for further details.

Thanks to our ongoing collaboration with Vattenfall, we have a continuos feedback from experts on the new problem specifications arising from new projects. In the present paper, we address new features of great importance, and show how they can be modeled and solved using MILP technology. In particular, we address three main extensions of the MILP model given in [6], that allow us to enforce a "closed loop" structure intended to handle possible cable failures, to penalize in a nonlinear way the number of cables entering each turbine, and to use the emergent Offshore Transformer Module technology [8] to replace substations.

The paper is organized as follows. The basic model proposed in [6] is briefly summarized in Subsection 2.1, while the three extensions are addressed in Subsections 2.2, 2.3 and 2.4, respectively. Section 3 finally reports computational results on a real case.

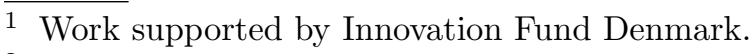

2 Email: martina.fischetti@vattenfall.com

3 Email: dapi@dtu.dk 


\section{MILP models}

We next describe the different MILP models we developed to consider different new trends in offshore cable routing.

\subsection{The basic MILP model}

We first need to briefly recall the basic model we developed for Vattenfall; the reader is refereed to [6] for fuller details.

Assuming that turbine positions have already been fixed, the problem of interest consists of finding an optimal cable connection between all turbines and the given substation(s), minimizing the total cable cost subject to the following requirements: (1) different cables, with different capacities and costs, are available; (2) the energy flow on each connection cannot exceed the capacity of the installed cable; (3) the energy flow leaving a turbine must be supported by a single cable; (4) cable crossing should be avoided; and (5) a given maximum number of cables, say $C$, can be connected to each substation;

Turbine positions can be viewed as the nodes of a complete and loop-free directed graph $G=(V, A)$, and all possible connections between them as directed arcs. Some nodes correspond to the substations that are considered as the roots of the distribution network, and are the only nodes that collect energy. The solution of the problem is a collection of directed trees rooted at the substations (arcs are directed toward the substation, following the orientation of the energy flow).

The model also allows for optional "Steiner" nodes, that can either be left uncovered, or have exactly one entering and one leaving cable. These dummy nodes are useful when considering obstacles in the area, or to allow for curvy connections between two nodes; see [6] for details.

Each node corresponds to a point in the plane, whose coordinates are used to compute distances between nodes as well as to determine whether two given line segments $[i, j]$ and $[h, k]$ cross each other, where $[a, b]$ denotes the line segment in the plane having nodes $a, b \in V$ as endpoints.

The nodeset $V$ is partitioned into $\left(V_{T}, V_{0}, V_{S}\right)$, where $V_{T}$ contains the nodes corresponding to the turbines, $V_{0}$ contains the nodes corresponding to the substation(s), and $V_{S}$ contains the Steiner nodes (if any). Furthermore, let $P_{h} \geq 0$ denote the power production at node $h \in V$, where $P_{h}>0$ for $h \in V_{T}$ and $P_{h}=0$ for $h \in V_{S}$ (nodes $h \in V_{0}$ corresponding to substations have $P_{h}=-1$ by convention).

Let $T$ denote the set of different cable types that can be used. Each cable 
type $t \in T$ has a given capacity $k_{t} \geq 0$ and a unit cost $u_{t} \geq 0$. Arc costs $c_{i, j}^{t}=u_{t} \cdot \operatorname{dist}(i, j)$ can then be computed for each arc $(i, j) \in A$ and for each cable type $t \in T$, where $\operatorname{dist}(i, j)$ is the Euclidean distance between nodes $i$ and $j$.

Decision variables are as follows. For each $\operatorname{arc}(i, j) \in A$, we have a continuous variable $f_{i, j} \geq 0$ representing the (directed) energy flow from $i$ to $j$, and a binary variable $x_{i, j}^{t}=1$ iff arc $(i, j)$ is constructed with cable type $t$ for each $t \in T$. Finally, binary variables $y_{i, j}=\sum_{t \in T} x_{i j}^{t}$ indicate whether an arc $(i, j)$ is built with any type of cable.

For the sake of space, we do not report the detailed MILP model here, and refer the reader to [6] for fuller details, and to Figure 1a for the plot of a typical solution.

\subsection{Closed-loop structure}

Generally speaking, turbines are designed to be connected to an electrical grid. Modern turbines are manufactured to have a certain autonomy to survive disconnected from the grid, but older models do not have this feature. This means that, in case of cable failures, the stand-alone turbines could suffer from major damages. In order to avoid this situation, parks with this kind of turbines need to have redundant cables in their layout. The main scope of these redundant cables is to keep all turbines connected to the grid, in case an inter-array cable failure occurs. When redundant cables are needed, a different cable-routing structure was required by our company partner, that we call closed-loop (or ring) structure. This structure consists in having at most one cable entering a turbine (i.e., a connection by string) and in pairing the leaf turbines by redundant cable connections; see Figure $1 \mathrm{~b}$ for an illustration. These connections always use only the cheapest cable available because they are intended to keep the turbines connected to the grid in case of a cable failure. The ring structure is the redundancy structure most used in practice, since it has high reliability and permits to easily locate failures [1]. As we will see in Section 3, the closed-loop structure can be significantly more expensive than the usual tree structure, so it should be imposed only if strictly required (i.e., if the park consists of old-technology turbines).

In order to impose the closed-loop structure, in our MILP model we introduce a new binary variable $q_{i, j}$ for each $(i, j) \in A$, where $q_{i, j}=1$ if a redundant cable has to be located between nodes $i$ and $j$. As the redundant cable connections have no orientation, we actually fix $q_{i, j}=0$ whenever $i \geq j$, thus halving the number of additional variable required. 
The new variables $q_{i, j}$ are then linked to the $y_{i, j}$ variables through the following constraints to be added to the basic MILP model:

$$
\begin{array}{cl}
\sum_{i \in V: i \neq h}\left(y_{i, h}+y_{h, i}+q_{i, h}+q_{h, i}\right)=2 \sum_{j \in V: j \neq h} y_{h, j}, & h \in V_{T} \cup V_{S} \\
q_{i, j} \quad=0, & (i, j) \in A: i \geq j \\
q_{i, j} \in\{0,1\}, & (i, j) \in A .
\end{array}
$$

Note that the degree-2 constraints (1) are not imposed for nodes $h \in V_{0}$ that correspond to substations (that are allowed to have degree 4 or more), and that the right-hand side term is zero in case node $h \in V_{S}$ is left uncovered. To avoid that the new arcs induce crossings in the final routing, in our branch-andcut solver we dynamically separate (for integer solutions only) the following extended no-cross constraints

$$
y_{i, j}+y_{j, i}+y_{h, k}+y_{k, h}+q_{i, j}+q_{j, i}+q_{h, k}+q_{k, h} \leq 1
$$

for each pair $[i, j]$ and $[h, k]$ of crossing edges.

As to the objective function, each new variable $q_{i, j}$ has a cost computed as $u_{\text {min }} \cdot \operatorname{dist}(i, j)$, where $u_{\text {min }}=\min _{t \in T} u_{t}$ is the unit cost of the least expensive cable.

\subsection{Branching penalties}

A branching structure is a layout where some turbines can have two or more entering cables. When allowed, this structure can lead to a considerable reduction in cable costs. However, one should also consider the extra cost incurred to connect multiple entering cables to the same turbine, as this configuration requires additional hardware (load breaker or disconnectors). In our study we considered turbines with at most two entering (and one leaving) cables. The standard package for the turbines includes connections for only one entering and leaving cable (this configuration has zero extra cost). If we want to have more than one entering cable (branching structure), we have to pay for the additional load breakers or disconnectors, and for the extra time to install them. Referring to our test-case park of Section 3, Vattenfall's experts estimated that the extra cost for having two $33 \mathrm{KV}$ cables entering the same turbine is $15 \mathrm{~K} €$ for the disconnector, plus $10 \mathrm{~K} €$ for installation. Note the the above extra costs (called branching penalties in what follows) depend on the number of arcs entering each turbine, but not in a linear way.

We next describe the modifications needed to deal with branching penalties in our MILP model. Let $k_{\max }$ be the maximum allowed in-degree for a node $v \in V_{T}$ (in typical applications, $k_{\max }=2$ or 3 ), and let $K=\left\{1, \cdots, k_{\max }\right\}$. 
Moreover, for each $k \in K$ let $\pi_{k}$ the extra-cost (penalty) incurred for each node in $V_{T}$ that has in-degree equal to $k$ in the final solution.

We introduce a new set of binary variables $z_{j}^{k}$ with $j \in V_{T}$ and $k \in K$, where $z_{j}^{k}=1$ iff the in-degree of node $j$ is equal to $k$. The new objective function then reads

$$
\min \sum_{(i, j) \in A} \sum_{t \in T} c_{i, j}^{t} x_{i, j}^{t}+\sum_{k \in K} \pi_{k} \sum_{j \in V_{T}} z_{j}^{k}
$$

while the basic MILP model is extended through the following additional constraints:

$$
\begin{aligned}
& \sum_{i \in V: i \neq j} y_{i, j}=\sum_{k \in K} k \cdot z_{j}^{k}, j \in V_{T} \\
& \sum_{k \in K} z_{j}^{k} \quad \leq 1, \quad j \in V_{T} \\
& z_{j}^{k} \quad \in\{0,1\}, \quad j \in V_{T} .
\end{aligned}
$$

Note that, in (6), we write " $\leq$ " instead of "=" so as to allow for leaf nodes with zero in-degree.

\subsection{Using Offshore Transformer Modules instead of substations}

Cable routing problems typically assume that substation(s) are fixed on input. In very recent years, however, companies are questioning about the need for offshore substations, that are big and expensive structures involving a lot of components - while only the main transformer is required in practice. In 2015, Siemens [8] proposed an innovative structure, called Offshore Transformer Module (OTM), that is able to handle the transformer function through a smaller and cheaper hardware to be attached directly to the turbine foundations. The turbines with this OTM structure can be connected directly to shore, or to other OTM structures, through so-called export cables. Export cables differ from inter-array cables, in that they operate at a different voltage and have a much higher capacity (and a much larger price). Due to their different voltage, export cables cannot be connected directly to inter-array cables, but require the installation of a transformer-hence the need of the OTM. See Figure 1d for an illustration of a typical cable routing involving export cables.

The first modification to our MILP model consists in introducing a single "dummy substation" associated with a node $s$ located on shore, that represents the connection to the bone electrical network. In addition, a special cable type $\tau$ is given on input, that corresponds to the export cable (with its capacity 
and unit cost). Also, we need to impose the following technical requirements: (a) no more than $\mu_{1}$ regular cables can enter a turbine, and (b) no more than $\mu_{2}$ export cables can enter each turbine. The above requirements can easily be modeled by the following additional constraints:

$$
\begin{aligned}
\sum_{i \in V: i \neq h} \sum_{t \in T: t \neq \tau} x_{i, h}^{t} \leq \mu_{1}, \quad h \in V_{T} \\
\sum_{i \in V: i \neq h} x_{i, h}^{\tau} \leq \mu_{2}, \quad h \in V_{T} .
\end{aligned}
$$

Finally, the fixed cost for each OTM can be added to the cost of each variable $x_{i, j}^{\tau}$ in the MILP objective function.

\section{Computational experiments}

The MILP-based heuristic presented in [6] can be applied to the new models without any substantial modification - thus confirming its flexibility.

For the sake of space, we report here some experiments on a single real wind farm site, namely Horns Rev 3. We compare the solutions found when using the various model versions addressed in the previous section. The possibility of quickly evaluating the economical impact of alternative design choices is considered of fundamental importance by the Vattenfall's engineers, who make several "what if" analyses before deciding the final cable routing to be implemented.

Horns Rev 3 is a big (350 MW) park in Denmark, still under construction. Fifty $8 \mathrm{MW}$ turbines are used in the layout. These are represented as black dots in the following plots. In this project, the offshore substation is given by the grid operator, thus its position and limits are fixed. At most 12 cables can be connected to the substation (red square in Figures 1a, 1b and 1c). Our set of cables consists of three types of cable: the black one supports 3 turbines at a cost of $393 € / \mathrm{m}$, the green one supports 4 turbines at a cost of $460 € / \mathrm{m}$, and the blue one supports 5 turbines at a cost of $540 € / \mathrm{m}$ (costs include both cable and installation costs). Our original model results in the layout of Figure 1a and does not consider other additional costs or constraints. The layout of Figure 1b is instead forced to use a closed-loop structure. This layout is $3.9 \mathrm{M} €$ more expensive than the previous one (including the cost for the redundant cables, in orange in the figure): this is the extra cost that the company needs to pay to ensure all turbines to be connected to the grid in case of inter-array cable failures. Figure 1c plots the layout obtained by considering the same constraints as in the basic test, but imposing that at most two cables 
can enter a turbine and that the extra cost for each of these branches is $25 \mathrm{~K} €$ (estimated by the company, considering the cost of the additional disconnector and installation). The solution is not really affected by these extra costs, and only slightly changes from Figure 1a. In the last test (Figure 1d) we were asked to evaluate the use of OTM structures instead of using the substation. Experts estimated a cost of $1 \mathrm{M} €$ for each OTM structure, and we imposed $\mu_{1}$ equal to 2 and $\mu_{2}$ equal to 1 . In this case the optimization considers also an extra cable (the export cable) that can support all turbines and has a cost of $1200 € / \mathrm{m}$. Figures 1 a to $1 \mathrm{~d}$ show the optimized layouts using our different models. The first three cases were solved to optimality in less than one hour, the last test was run with a time-limit of 10 hours. Table 1 reports a few statistics about the runs.

Table 1

Computationa information about the runs

\begin{tabular}{|lccrrrr|}
\hline Optimization mode & $\begin{array}{c}\text { LP bound } \\
{[\mathrm{M} €[}\end{array}$ & $\begin{array}{r}\text { best sol } \\
{[\mathrm{M} €]}\end{array}$ & gap & nodes & $\begin{array}{r}\text { no-cross } \\
\text { constr. }\end{array}$ & $\begin{array}{r}\text { final time } \\
(\mathrm{sec})\end{array}$ \\
\hline original model & 36.69 & 36.69 & 0 & 4964 & 137 & 176.41 \\
closed-loop & 40.63 & 40.63 & 0 & 96649 & 972 & 2931.90 \\
branch penalties & 36.74 & 36.74 & 0 & 596 & 19 & 232.52 \\
OTMs & 60.89 & 69.82 & 12.79 & 389452 & 5076 & 36000 \\
\hline
\end{tabular}

The costs reported in Table 1 are the cost computed with the specific set-up specified in the first column (as they come out from the optimization model). Notice that, because of the extra branch costs or the OTM related costs, it would be incorrect to compare the "branching penalties" or the "OTMs" solutions with the "original model" using directly the costs of Table 1. We can correctly estimate the savings obtained by including the branching costs in the model in the following way: we re-evaluate the cost of the original model solution adding $25 \mathrm{~K}$ €for each branching in the solution. The solution obtained with the original model is $0.02 \mathrm{M} €$ more expensive than the "branching optimized" one. We can estimate the savings obtained by using OTMs instead of a substation in the following way: we fix the basic layout of Figure 1a and we add the cost for the export cable to shore (computed as $1200 € / \mathrm{m}$ ) and the substation cost of $100 \mathrm{M} €$. In this example case, the savings are about $101 \mathrm{M} €$. 


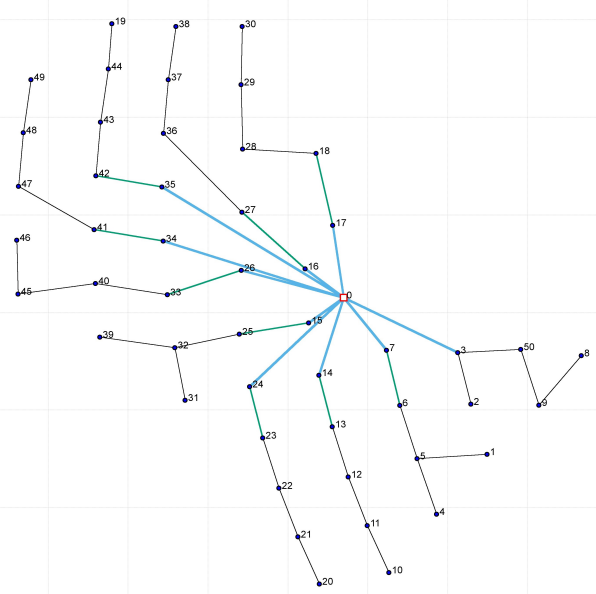

(a) Original model

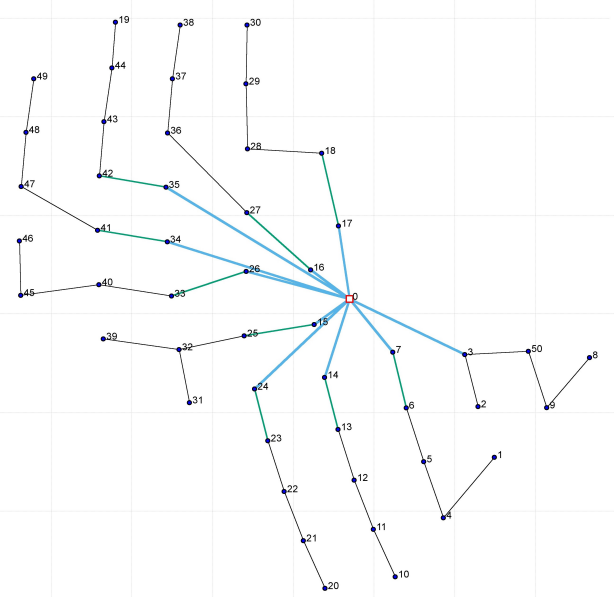

(c) Branching penalties

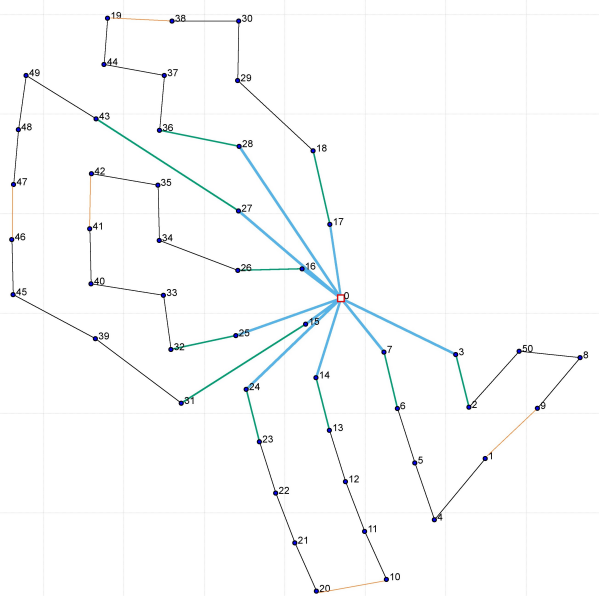

(b) Closed-loop structure

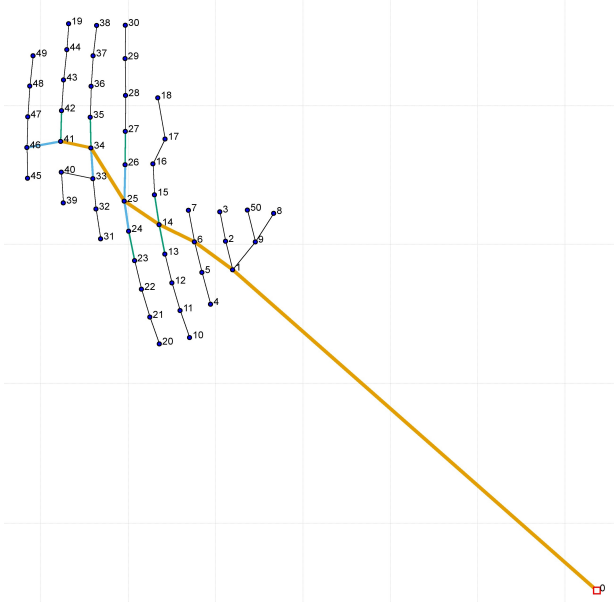

(d) Using OTMs

Fig. 1. Solutions using our different models.

\section{Conclusions}

In the present paper we used Mixed Integer Linear Programming techniques to solve new versions of the classical offshore wind farm cable routing problem. Thanks to the close collaboration with Vattenfall AB Wind, we have been able to investigate the most recent trends on the market and evaluate their impact on the cable routing.

Turbines are becoming more customized, allowing them to survive disconnected from the grid in case of failures, or even to substitute substations 
through the so-called Offshore Transformer Modules. Turbine customization opens up for new possibilities in the park layout. Therefore it is crucial to have an optimization tool able to quickly evaluate their impact on the wind park costs. In the present paper we introduced a flexible and reliable tool, that scales well for bigger parks and more complex constraints. We have been able to handle new features in the model (i.e. closed-loop structure, non-linear branching penalties and OTMs) and to quantify their effect on a real-case. The outcome of our tests indicates that millions of euros are involved in these analyses, so decisions based on optimized solutions can lead to substantial savings.

\section{References}

[1] A. Sannino, H. B. and E. Nielse, Reliability of collection grids for large offshore wind parks, Proceedings of 9th International Conference on Probabilistic Methods Applied to Power Systems (2006).

[2] Bauer, J. and J. Lysgaard, The offshore wind farm array cable layout problem: a planar open vehicle routing problem, Journal of the Operational Research Society 66 (2015), pp. 360-368.

[3] Berzan, C., K. Veeramachaneni, J. McDermott and U. O. Reilly, Algorithms for cable network design on large-scale wind farms, Tech. Rep. Tufts University (2011).

[4] Dutta, S., "Data Mining and Graph Theory Focused Solutions to Smart Grid Challenges," Master's thesis, University of Illinois (2012).

[5] Fagerfjall, P., "Optimizing Wind Farm Layout - More Bang for the Buck Using Mixed Integer Linear Programming," Master's thesis, Department of Mathematical Sciences, Chalmers University of Technology and Gothenburg University, Goteborg, Sweden (2010).

[6] Fischetti, M. and D. Pisinger, Optimizing wind farm cable routing considering power losses, submitted for publication and available at http://orbit.dtu.dk/ws/files/126231483/TechRep_FischettiPisinger.pdf (2016).

[7] Hertz, A., O. Marcotte, A. Mdimagh, M. Carreau and F. Welt, Optimizing the design of a wind farm collection network, INFOR 50 (2012), pp. 95-104.

[8] Siemens, New AC Grid Access Solution from Siemens: Lighter, faster, cheaper, http://www.siemens.com/press/en/pressrelease/?press=/en/ pressrelease/2015/energymanagement/pr2015030151emen.htm. 